\title{
The complex alteration in the network of IL-17-type cytokines in patients with hereditary angioedema
}

\author{
Francesco Arcoleo ${ }^{1}\left(\right.$ ) Mariangela Lo Pizzo ${ }^{1} \cdot$ Gabriella Misiano $^{2} \cdot$ Salvatore Milano ${ }^{2}$. \\ Giuseppina Colonna Romano ${ }^{2} \cdot$ Vito Muggeo $^{3} \cdot$ Enrico Cillari $^{4}$
}

Received: 9 October 2017 / Accepted: 15 March 2018 / Published online: 6 April 2018

(c) Springer International Publishing AG, part of Springer Nature 2018

\begin{abstract}
Hereditary angioedema (HAE) is a rare autosomic-dominant disorder characterized by a deficiency of C1 esterase inhibitor which causes episodic swellings of subcutaneous tissues, bowel walls and upper airways that are disabling and potentially life-threatening. We evaluated $n=17$ patients with confirmed HAE diagnosis during attack and remission state and $n=19$ healthy subjects. The samples were tested for a panel of IL (Interleukin)-17-type cytokines (IL-1 $\beta$, IL-6, IL-10, granulocyte-macrophage colony stimulating factor (GM-CSF), IL-17, IL-21, IL-22, IL-23) and transforming growth factor-beta (TGF- $\beta$ ) subtypes. Data indicate that there are variations of cytokine levels in HAE subjects comparing the condition during the crisis respect to the value in the remission phase, in particular type 17 signature cytokines are increased, whereas IL-23 is unmodified and TGF- $\beta 3$ is significantly reduced. When comparing healthy and HAE subjects in the remission state, we found a significant difference for IL-17, GM-CSF, IL-21, TGF- $\beta 1$ and TGF- $\beta 2$ cytokines. These results confirm and extend our previous findings indicating that in HAE there is operating an inflammatory activation process, which involves also $\mathrm{T}$ helper 17 (Th17) cytokines and TGF- $\beta$ isoforms, associated with localized angioedema attacks and characterized by elevated bradykinin levels.
\end{abstract}

Keywords Hereditary angioedema $\cdot \mathrm{C} 1$ esterase inhibitor $\cdot$ Cytokines

\section{Introduction}

Hereditary angioedema (HAE) is a rare autosomic-dominant disorder characterized by a deficiency of $\mathrm{C} 1$ esterase inhibitor (C1INH) which causes episodic swellings of subcutaneous tissues, bowel walls and upper airways that are disabling and potentially life-threatening [1]. C1INH has a regulatory role in the classical complement pathway, in the coagulation cascade, fibrinolysis and kallikrein-kinin system $[1,2]$. Indeed C1INH is the major inhibitor of the early

Francesco Arcoleo

francescorcoleo@alice.it

1 Patologia Clinica Presidio Ospedaliero V. Cervello, Ospedali Riuniti Villa Sofia-Cervello, Palermo, Italy

2 Dipartimento di Biopatologia e Biotecnologie Mediche (DIBIMED), University of Palermo, Palermo, Italy

3 Dipartimento Scienze Economiche, Aziendali e Statistiche, University of Palermo, Palermo, Italy

4 Dipartimento Immunologia Allergologia, Istituto Medico Europeo (ISME), Palermo, Italy complement proteases $\mathrm{C} 1 \mathrm{r}$ and $\mathrm{C} 1 \mathrm{~s}$ and contact system proteases plasma kallikrein and coagulation factor XIIa and to a lesser degree of plasmin and factor XIa. Its reduced activity may result in an elevated plasma levels of bradykinin [2-4], the key mediator of symptoms in HAE [3, 4]. It has become widely accepted that during HAE attacks permeability is increased by binding of bradykinin to bradykinin receptor type 2 (BKR-2) and possibly to BKR-1 activating signalling cascades resulting in production of vasodilating mediators. In fact, C1INH moderates the conversion of prekallikrein to kallikrein, reducing in such a way the kallicrein-induced bradykinin activaction [4]. In HAE, the activation of contact system leads to the generation of bradykinin that mediates the disease and local process [5-7]. However, the activation and the swelling often occur at multiple sites during the attack [8] preceding in several patients, muscle aches, rash and fatigue $[9,10]$. Altogether, the C1INH deficiency causes an enhancement of mediators which induce an increase in capillary permeability responsible of recurrent, non-pruritic, attack of angioedema. The etiopathogenic process leading to the different clinical signs is not completely understood. 
Various cells of the immune system seem to be involved [11], and C1INH synthesis is subject to complex positive and negative control by cytokines [12-15]. In fact, recombinant IFN $\gamma$ and to the lesser extent GM-CSF, tumour necrosis factor-alpha (TNF $\alpha$ ) and IL-6 regulate C1INH synthesis in various cell types $[12,13]$.

We have previously observed a constant increase in IL-17, GM-CSF and fibroblast growth factor-beta (FGF $\beta$ ) in the peripheral blood of HAE patients during the acute attack and also in the remission phase between the crises, compared with healthy controls $[14,15]$. These data indicated that raised levels of some cytokines could have a relation with the increase in bradykinin in more sites, which has induced some researches to hypothesize a systemic activation process of the contact system as precipitating event $[16,17]$.

IL-17 together with GM-CSF, IL-21, IL-22, IL-23 and IL- 6 are produced by Th17 and type 17 cells $(\gamma \delta \mathrm{T}$ cells, natural killer cells, NKT cells and innate lymphoid cellsILCs) under a peculiar cytokine milieu favouring their generation [18-20]. Effectively, the generation of Th17 cells is activated by TGF- $\beta$ and IL- 6 , which initiate the commitment of a naïve Th cells to the Th17 lineage [21-23]. These cells produce IL-21, a member of the $\gamma c$ chain family of cytokines, that plays a key amplification role in the generation of Th17 cells functioning in an autocrine manner to reinforce the commitment of newly differentiating Th17 cells towards the Th17 lineage [24-26]. Furthermore, the maintenance and the expansion of Th17 cells are led by another cytokine produced mainly by macrophages, IL-23 [25, 27 , 28 , that together with IL-1 $\beta$ induces local tissue inflammation [27, 28]. Finally, terminally differentiated Th17 cells, in response to IL-23, produce IL-22, which is important in the interaction with various tissue cells $[29,30]$. On the other hand, the stimulation of Th17 cells and type 17 cells with IL-1 $\beta$ and IL-23 induces local inflammation which is mediated by IL-17, IL-22 and GM-CSF [28, 31]. It is particularly relevant that Th17 can be host protective, expressing both IL-17 and IL-10, or highly inflammatory expressing IL-17, IL-22, IFN $\gamma$ and GM-CSF [28, 31-33].
All the above data, together with our observation of high production of some type 17 signature cytokines such as IL-17 and GM-CSF $[14,15]$ in HAE patients, have led us to extend previous studies analysing also the modification in IL-23, IL-17, IL-21, IL-22 and TGF $\beta$ subtypes in blood samples from individuals with HAE both during the onset of crisis and in the asymptomatic period compared with healthy subjects.

\section{Methods}

\section{Study design}

We evaluated a total of $n=17$ patients with HAE (11 female, 6 male; ages 9-65 old years). The patients enlisted in this study consisted in subjects affering to the Angioedema Center in the V. Cervello Hospital, Palermo. The control group consisted of $n=19$ apparently healthy subjects (10 females and 9 males; ages 10-54 years old); no one of them or of their family members ever had oedematous attack, as ascertained by accurate anamnesis. Main characteristics of the samples, cases and controls, are summarized in Table 1, along with the ranges of all cytokines studied. None of the selected subjects was affected by neoplastic, infectious or autoimmune diseases, or received any drug influencing immune functions at the time of the study. For patients with HAE, 2-8 repeated measurements for each subject were available, with a different number of observation between attacks and remissions phases. Repeated observations were also collected for healthy individuals. The total numbers of observations are 80 for cases and 39 for the controls. The study protocol was approved by the local ethic committees and it conforms to the provisions of the Declaration of Helsinki in 1995 (as revised in Edinburgh, 2000). To avoid interferences with cytokine assay determination, we excluded patients with the acute attacks if they have received plasma-derived $\mathrm{C} 1$ esterase inhibitor for a crisis occurred in the three previous days. We also excluded

Table 1 Frequency distribution (A), mean and standard deviation (B) of the variable age in the study sample $(n=17$ patients with confirmed HAE) and in the control group $(n=19)$ according to the gender

\begin{tabular}{lcr}
\hline & Study sample & Control group \\
\hline$A$ & & $10(53 \%)$ \\
Female & $11(64.71 \%)$ & $9(47 \%)$ \\
Male & $6(35.29 \%)$ & $19(100 \%)$ \\
Total & $17(100 \%)$ & SD \\
\hline & Mean & 16.6 \\
Study sample & & 10.3 \\
Control group & 35.5 & 39.5 \\
\hline
\end{tabular}


patients receiving tranexamic acid within a week before the new crisis or the patients treated with angiotensin converting enzyme (ACE) inhibitors for hypertension or pregnant patients. HAE subjects presented cutaneous or abdominal attacks and were asked to evaluate the severity of three specific symptoms (cutaneous swelling, cutaneous pain and abdominal pain) using a visual analog scale (VAS). For each symptom, patients drew a vertical line on an ungraduated $100-\mathrm{mm}$ VAS scale, according to severity $(0 \mathrm{~mm}-$ no symptoms; $100 \mathrm{~mm}$-worst possible severity). Those with a VAS score $>30 \mathrm{~mm}$ for one or more of these three symptoms, and no exclusion criteria, were considered to have an eligible attack.

Each subject underwent a fasting blood sampling. Serum samples were separated from blood within $30 \mathrm{~min}$ of venipuncture using 6-ml vacuette with serum clot activator (Greiner Bio-One GmbH, Kremsmunster, Austria), by clotting and centrifugation at $400 \mathrm{~g}$ for $10 \mathrm{~min}$. Samples were aliquoted and stored at $-80{ }^{\circ} \mathrm{C}$ until testing. For plasma studies, samples were collected in 3-ml vacuette K3EDTA (Greiner Bio-One), immediately centrifuged at $1800 \mathrm{rpm}$ for $15 \mathrm{~min}$ to isolate cell-free plasmas that were immediately stored at $-80{ }^{\circ} \mathrm{C}$.

\section{Evaluation of $\mathrm{C4}$, $\mathrm{C}$ IINH}

Diagnosis was confirmed by the medical history and a finding of level of antigenic (type I) or functional (type II) C1 esterase inhibitor of less than half of normal levels. Normal C1-INH plasma values range in our laboratory is between 15 and $35 \mathrm{mg} / \mathrm{dL}$ for antigenic and 70 and $130 \%$ for functional (2.5 and 97.5 percentile) of normal pool. For those symptomatic patients without evidence of C1-INH deficiency within the family, the genetic origin of the disease was confirmed by the evidence of mutation in C1-INH gene (SERPING1). Functional C1-INH was measured using a chromogenic assay (Technochrom C1-inhibitor, Technoclone $\mathrm{GmbH}$, Vienna, Austria), C1-INH antigen and $\mathrm{C} 4$ antigens by rate nephelometry using the Immage Immunochemistry System (Beckman Coulter, Fullerton, CA).

\section{Assay for cytokine determination by luminex or ELISA}

Immediately before the cytokine assay, thawed serum samples were centrifuged at 12,000 rpm for 5-10 min to allow precipitation of any lipids excess, that may interfere with subsequent analysis. The samples were tested for a panel of all cytokines (IL-6, IL-10, IL-13, IFN $\gamma$ GM-CSF, IL-17, IL-21, IL-22, IL-23, TGF $\beta$ subtypes), using Bio-plex kit (BioRad, Milan, Italy), following manufacturer instructions. The assay was carried out using the Luminex system, based on the measurement of fluorescent signals released by a suspension of microspheres bringing immobilized multiplex cytokine-specific antibodies in 96-well plates. The combination of fluorimetric signal of microspheres with that released by a secondary antibody allows to measure cytokine concentration-related signals converted by a processor. Briefly, 50 $\mu \mathrm{L}$ of samples diluted 1:4 in a dilution buffer were incubated at room temperature in the presence of beads conjugated with specific antibodies for the different cytokines. After a wash to remove the excess of not bound serum components, an incubation with the biotin-conjugated secondary antibodies was performed. Finally, after another washing step and the streptavidin-PE complex addition, the fluorimetric signal, by using the luminex plate reader, was detected. The assay was performed using a 8-point standard curve for every cytokine. Samples were analysed on a Luminex 100 device (BioRad, München, Germany), and the data were evaluated using the Bio-Plex Manager software (BioRad). Standards, internal controls and samples were reported as means of duplicate measurements. We considered zero value when the level of mediators was under the detection limit.

\section{Statistical analysis}

Cytokines levels were modelled using linear mixed models (LMM), namely regression models with fixed and random effects [22]. Random effects account for the unbalanced and correlated repeated measurement within the same subject, and fixed effects quantify the difference in the average levels of cytokines between remission versus crisis state. The LMM framework is able to detect possible differences due to factors while accounting for intra-subject correlation and physiological variability. Also, due to detection limits of some cytokines (Tab 2), the response values were considered censored at the observed LOD value. We fitted separate

Table 2 Limits of detection (LOD) and relevant percentages of measurements falling below for each outcome variable

\begin{tabular}{llc}
\hline & LOD $(\mathrm{pg} / \mathrm{ml})$ & $\%<\mathrm{LOD}$ \\
\hline IL6 & 0.67 & 0 \\
IL10 & 0.30 & 84.3 \\
IL17 & 0.28 & 12.4 \\
GM-CSF & 2.2 & 16.8 \\
IL1- $\beta$ & 0.02 & 89.9 \\
IFN- $\gamma$ & 0.43 & 0 \\
IL21 & 2.13 & 31.0 \\
IL22 & 0.3 & 95.7 \\
IL23 & 1.55 & 18.1 \\
TGF- $\beta 1$ & 3.9 & 2.6 \\
TGF- $\beta 2$ & 1.9 & 3.45 \\
TGF- $\beta 3$ & 0.5 & 48.3 \\
\hline
\end{tabular}


LMM for each cytokine outcome and each model always included gender and age as confounders.

\section{Results}

\section{Demographic data and principal characteristics}

In Table 1A, the frequency distribution of study samples (patients with confirmed HAE) and control group according to gender is presented, which indicates that there are no significant differences. Table $1 \mathrm{~B}$ confirms that the mean age of controls and patients is similar.

\section{Cytokine data}

Characteristics of cytokine levels ( $\min$ and max) in the observed samples (cases and healthy subjects) and detection limit (LOD) for cytokines are reported in Table 2. Figure 1 shows boxplots of the distributions of IL-6, IL-10, IL-17, GM-CSF, IL-21, IL-22, IL-23, TGF $\beta 1$, TGF $\beta 2$ and TGF $\beta 3$ cytokines in HAE subjects at both remission and crisis state. The rise of all these cytokines during the acute attacks compared with the remission values suggests that type 17 signature cytokines (IL-17, GM-CSF) and the stimulator of Th17 and type 17 cells (IL-21, IL-6, TGF $\beta$ ) are modified in this disease. For IL-22, and IL1 $\beta$, since we observed in unusual boxplots due to high number of censored measurements (high number of values near to the limit detection) (Table 2), we retain not possible any explanation on the differences between the two groups. Broadly speaking, the higher values for the medians in the 'crisis' group suggest that cytokine levels are increased during the attack. On the top of each panel in the same Fig. 1, the values relevant to healthy subjects are reported in terms of mean standard deviation. It appears that IL-17, GM-CSF, IL-21, IL-23, TGF $\beta 1$, TGF $\beta 2$ and TGF $\beta 3$ are below the mean value of them in HAE patients in the remission phase. Table 3 reports parameter estimates from the linear mixed models fitted for each cytokine outcome by restricted maximum likelihood. Although each model always included gender, age and random effects for the intercept to account for possible correlation among measurements of the same subjects, we report estimates only for the average increase in cytokine levels during 'crisis' with respect to their amount in the same patients without crisis. Out of the 15 analysed cytokines, we found out significant ( $p$ value $<0.05$ ) differences between crisis and remission state for seven cytokines: IL-6, IL-10, IL-17, IL-21, GM-CSF, TGF $\beta 1$ and TGF $\beta 2$. In detail, in the case of IL- 6 the rise was $4.6 \mathrm{pg} / \mathrm{ml}$, for IL-10 $2.48 \mathrm{pg} / \mathrm{ml}$, for IL-17 $10.5 \mathrm{pg} / \mathrm{ml}$, for IL-21 $25.2 \mathrm{pg} /$ $\mathrm{ml}$, for GM-CSF $14.1 \mathrm{pg} / \mathrm{ml}$, for TGF $\beta 129,4 \mathrm{pg} / \mathrm{ml}$ and for TGF $\beta 249,4 \mathrm{pg} / \mathrm{ml}$ higher in crisis condition respect to the basal value. IL-10 was the only clearly defined anti-inflammatory cytokine increased during the crisis $(p=0.0002)$ in these HAE patients (Table 3). On the contrary, TGF $\beta 3$ was the only mediator that presented an extremely significant decrease during the crisis respect to the value in the remission state. IL-22 was not modified in both the two observation phases. In same patients, the increases in all different cytokines were also detectable.

\section{Discussion}

In this study, comparing angioedema patients before and after the acute crisis with matched control subjects, we observed several significant modifications of Th17 lymphokine network. In the patients before the onset of crisis, the most prominent up-regulated cytokines were GM-CSF, IL-17, IL-21, TGF $\beta 1$ and TGF $\beta 2$. The same cytokines, plus IL-6, were further increased during the onset of the crisis. Data endorse the role of IL-17 pathway $[34,35]$ in this disease, working as triggering mediators. IL-17 maintains the activation of cytokine pathway, since it was always high following the onset of crisis, together with GM-CSF, IL-21 and IL-6. Since Th17 can be host protective, expressing both IL-17 and IL-10, or highly inflammatory expressing IL-17, IL-22, IFN $\gamma$ and GM-CSF [28, 31, 32, 36], it is possible that the ultimate Th17 activity is just determined by the balance of these effector functions. On the other hand, Th17 cells activated by TGF $\beta$ and IL- 6 promote mucosal defence and inhibition of immunopathogenic responses [32, 33 ] whereas IL-23 promotes the development of pathogenic Th17 cells [37, 38]; therefore, in HAE the normal level of IL-23 at baseline and during acute attacks could not evolve Th17 cells to cause autoimmunity, but to promote effective host defence. The not significant increase in IL-23 during the crisis could be one of the various mechanism induced to moderate inflammation, as happened for IL-10 that it is involved in the inhibition of excessive inflammatory response [39-41] and together with TGF $\beta$ could activate regulatory $\mathrm{T}$ (Treg) cells to moderate the high Th17 activation [21, 41, 42]. In agreement with that is the absence in the literature of data indicating an increase in autoimmune diseases in HAE, even though they have high levels in IL-17, that could be explained by the above protective Th17 subset activated by IL-10, and by the environmental reduction in IL-22, TGF $\beta 3$, associated with unmodified levels of IL-23. The latter observation in HAE patients is extremely interesting because TGF $\beta 3$ promotes the development of inflammatory and pathogenic Th17 by upregulating IL-23 receptor expression and subsequent exposure to IL-23, essential for the maturation of inflammatory Th17 cells [35].

The high rise in IL-17 together with the other cytokines strongly allows us to conjecture that HAE cannot be 

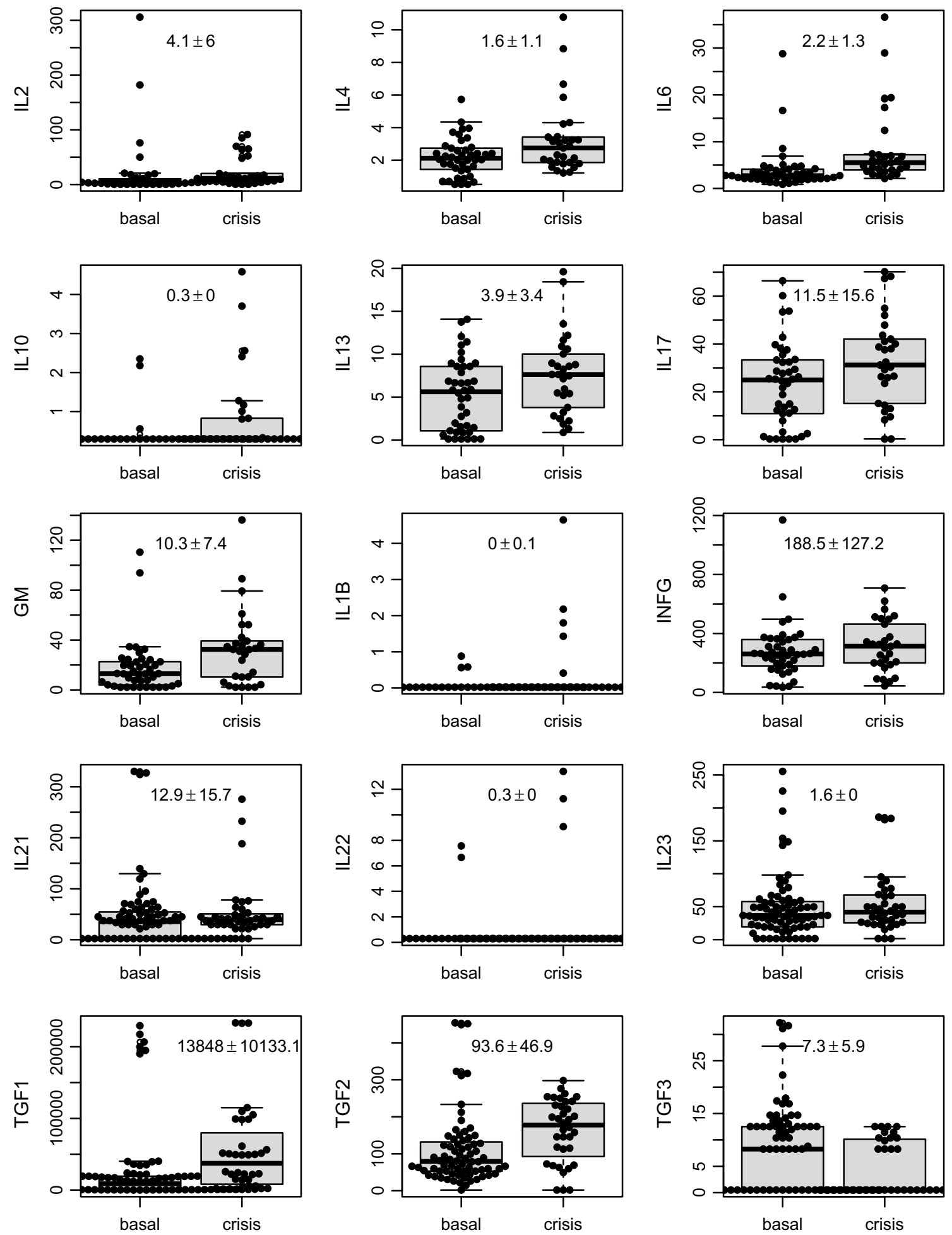

Fig. 1 Boxplots of the distributions of IL-1 $\beta$, IL-2, IL-4, IL-6, IL-10, IL-13, IL-17, GM-CSF, IL-21, IL-22, IL-23, TGF $\beta 1$, TGF $\beta 2$ and TGFß3 cytokines in HAE subjects at both basal and crisis state.

considered simply as a disease characterized by a bradykinin-mediated angioedema caused by the interaction with BKR type 2 (BKR-2) [3, 43], but a more complex event
On the top of each panel, the values relevant to healthy subjects are reported in terms of mean \pm standard deviation

with local generation of bradykinin in more sites and local expression of both BKR-1 and BKR-2 associated with circulating increase in cytokines [16]. BKR-1 is exposed on 
Table 3 Estimated average differences (with SE and $p$ values) 'crisis versus remission' from random effects models accounting for censoring and repeated measurements

\begin{tabular}{lccc}
\hline & Est & SE & $p$ value \\
\hline IL6 & 4.60 & 1.14 & 0.0002 \\
IL10 & 2.48 & 0.65 & 0.0002 \\
IL17 & 10.5 & 4.18 & 0.0118 \\
GM-CSF & 14.1 & 4.19 & 0.0008 \\
IL1- $\beta$ & 2.29 & 0.97 & 0.0179 \\
IFN- $\gamma$ & 31.9 & 38.3 & 0.41 \\
IL21 & 25.2 & 13.1 & 0.0551 \\
IL22 & 11.2 & 7.31 & 0.13 \\
IL23 & 6.65 & 7.58 & 0.38 \\
TGF- $\beta 1$ & 29.4 & 8718 & $<0.0001$ \\
TGF- $\beta 2$ & 49.4 & 17.8 & 0.0055 \\
TGF- $\beta 3$ & -6.69 & 2.40 & 0.0053 \\
\hline
\end{tabular}

endothelial cells on stimulation with kinins and cytokines [44-46], and its involvement in the pathogenesis of HAE attacks might be just suggested by increase in inflammatory cytokines and by often increased plasma $\mathrm{C}$ reactive protein levels, during severe HAE attacks [46], which reflect cytokine production in vivo. These circulating mediators have induced some authors to postulate a systemic activation process in the precipitating events $[16,17]$.

Furthermore, it has been described a protective role of bradykinin in experimental autoimmune encephalomyelitis, where the increased level of bradykinins induced by ACE inhibitors compounds inhibited the migration of IL23-activated pathogenic Th17 lymphocytes in the sites of inflammation in the brain [47]. This could take account of the angioedema characteristics in HAE patients, which by definition is self-limiting.

Even though it is possible to postulate that the higher levels of cytokines could be secondary to the effusion observed during the attacks with consequent haemoconcentration, it is not sustainable in the case of the high levels of IL-17, IL-21, GM-CSF, TGF $\beta 1$ and TGF $\beta 2$ in basal conditions and in the absence of crisis. Studies are in progress to evaluate the modifications of Th17 and Th17-type subsets in various phases of the diseases and in the various forms of HAE and to correlate them with cytokine assessment and their polymorphism.

In conclusion, this study confirms, extending our previous data $[14,15]$, that in HAE subjects there is a cytokine milieu favouring expansion of Th17 or Th17-type subsets capable of producing cytokines that are associated with contact activation by bradykinin leading to local angioedema formation responsible of increase in capillary permeability and subcutaneous swelling $[5,15,47]$. The expansion of non-pathogenic Th17 could down-modulate inflammatory response favouring the natural resolution of angioedema [28, 31-33]. These complex alterations in the cytokine network in HAE can bring in the future novel therapeutic approaches considering that nowadays cytokines responsible for regulation of a wide range of processes during development of the normal immune response are among the most successful therapeutic targets.

Funding This study was funded by Hospital funds.

\section{Compliance with ethical standards}

Conflict of interest The authors declare that they have no conflict of interest.

Ethical approval All procedures performed in studies involving human participants were in accordance with the ethical standards of the institutional and/or national research committee and with the 1964 Helsinki Declaration and its later amendments or comparable ethical standards. This article does not contain any studies with animals performed by any of the authors.

Informed consent Informed consent was obtained from all individual participants included in the study.

\section{References}

1. Agostoni A, Cicardi M. Hereditary and acquired C1-inhibitor deficiency: biological and clinical characteristics in 235 patients. Med (Baltimore). 1992;71(4):206-15.

2. Cicardi M, Banerji A, Bracho F, et al. Icatibant, a new bradykininreceptor antagonist, in hereditary angioedema. $\mathrm{N}$ Engl $\mathrm{J}$ Med. 2010;363(6):532-41.

3. Nussberger J, Cugno M, Amstutz C, Cicardi M, Pellacani A, Agostoni A. Plasma bradykinin in angio-oedema. Lancet. 1998;351:1693-7.

4. Cugno M, Nussberger J, Cicardi M, Agostoni A. Bradykinin and the pathophysiology of angioedema. Int Immunopharmacol. 2003:3:311-7.

5. Longhurst $\mathrm{H}$, Cicardi M. Hereditary angioedema. Lancet. 2012;379:474-81.

6. Zuraw B. Hereditary angioedema. N Engl J Med. 2008;359:1027-36.

7. Kusuma A, Relan A, Knulst AC, et al. Clinical impact of peripheral attacks in hereditary angioedema patients. Am J Med. 2012;125:937.e17-24.

8. Hofman ZLM, Relan A, Hack CE. Hereditary Angioedema attacks: local swelling at multiple sites. Clin Rev Allergy Immunol. 2016;50:34-40.

9. Prematta MG, Kemp JG, Gibbs JG, Mende C, Rhoads C, Craig TJ. Fequency, timing, and type of prodromal symptoms associated with ereditary angioedema attacks. Allergy Asthma Proc. 2009;30:506-11.

10. Magerl M, Doumoulakis G, Kalkounou I, et al. Characterization of prodromal symptoms in a large population of patiets with hereditary angioedema. Clin Exp Dermatol. 2014;39:298-303.

11. Cillari E, Misiano G, Aricò M, et al. Modification of peripheral blood T-lymphocyte surface receptors and Langerhans cell numbers in hereditary angioedema. Am J Clin Pathol. 1986;85(3):305-11. 
12. Prada AE, Zahedi K, Davis AE. Regulation of C1 inhibitor synthesis. Immunobiology. 1998;199(2):377-88 (Review).

13. Gluszko P, Undas A, Amenta S, Szczeklik A, Schmaier AH. Administration of gamma interferon in human subjects decreases plasminogen activation and fibrinolysis without influencing $\mathrm{C} 1$ inhibitor. J Lab Clin Med. 1994;123(2):232-40.

14. Arcoleo F, Salemi M, La Porta A, et al. Upregulation of cytokines and IL-17 in patients with hereditary angioedema. Clin Chem Lab Med. 2014;52(5):e91-3.

15. Salemi M, Mandalà V, Muggeo V, et al. Growth factors and IL-17 in hereditary angioedema. Clin Exp Med. 2016;16(2):213-8.

16. Hofman ZLM, Relan A, Zeerleder S, Drouet C, Zuraw B, Hack CE. Angioedema attacks in patients with hereditary angioedema: local manifestations of a systemic activation process. J Allergy Clin Immunol. 2016;138:359-66.

17. Berrettini M, Lammle B, White T, et al. Detection of in vitro and in vivo cleavage of high molecular weight kininogen in human plasma by immunoblotting with monoclonal antibodies. Blood. 1986;68:455-61.

18. Cua DJ, Tato CM. Innate IL-17 producing cells: the sentinels of the immune system. Nat Rev Immunol. 2010;10:479-89.

19. Zuniga LA, Jain R, Haines C, Cua DJ. Th17 cell development: from the cradle to the grave. Immunol Rev. 2013;252:78-88.

20. Marks BR, Nowyhed HN, Choi JY, et al. Thymic self-reactivity selects natural interleukin 17-producing cells thet can regulate peripheral inflammation. Nat Immunol. 2009;10:1125-32.

21. Romagnani S. Human Th17 cells. Arthr Res Therapy. 2008;10(2):206 (Review).

22. Acosta-Rodriguez EV, Rivino L, Geginat J, et al. Surface phenotype and antigenic specificity of human interleukin 17-producing T helper memory cells. Nat Immunol. 2007;8(6):639-46.

23. McGeachy MJ, Bak-Jensen KS, Chen Y, et al. TGF-beta and IL- 6 drive the production of IL-17 and IL-10 by T cells and restrain $\mathrm{T}(\mathrm{H})-17$ cell-mediated pathology. Nat Immunol. 2007;8(12):1390-7.

24. Korn T, Bettelli E, Oukka M, Kuchroo VK. IL-17 and Th17 Cells. Annu Rev Immunol. 2009;27:485-517 (Review).

25. Wilke CM, Bishop K, Fox D, Zou W. Deciphering the role of Th17 cells in human disease. Trends Immunol. 2011;32(12):603-11.

26. Korn T, Bettelli E, Gao W, et al. IL-21 initiates an alternative pathway to induce proinflammatory TH17 cells. Nature. 2001;448:484-7.

27. Cua DJ, Sherlock J, Chen Y, et al. Interleukin-23 rather than interleukin-12 is the critical cytokine for autoimmune inflammation of the brain. Nature. 2003;421:744-8.

28. Langrish CL, Chen Y, Blumenschein WM, et al. IL-23 drives a pathogenic $\mathrm{T}$ cell population that induces autoimmune inflammation. J Exp Med. 2005;201:233-40.

29. Liang SC, Tan XY, Luxenberg DP, et al. Interleukin (IL)-22 and IL-17 are coexpressed by Th17 cells and cooperatively enhance expression of antimicrobial peptides. J Exp Med. 2006;203:2271-9.

30. Alam MS, Maekawa Y, Kitamura A, et al. Notch signaling drives IL-22 secretion in CD4+ T cells by stimulating the aryl hydrocarbon receptor. Proc Natl Acad Sci USA. 2010;107:5943-8.
31. Zheng Y, Danilenko DM, Valdez P. Interleukin-22, a TH17 cytokine, mediates IL-23-induced dermal inflammation and acanthosis. Nature. 2007;445:648-51.

32. McGeachy MJ, Bak-Jensen KS, Chen Y, et al. TGF-beta and IL-6 drive the production of IL-17 and IL-10by T cells and restrain Th17 cell-mediated pathology. Nat Immunol. 2007;8:1390-7.

33. Esplugues E, Huber S, Gagliani N, et al. Control of Th17cells occurs in the small intestine. Nature. 2011;465:514-8.

34. McGeachy MJ, Chen Y, Tato CM, et al. The interleukin 23 receptor is essential for the terminal differentiation of interleukin 17-producing effector T helper cells in vivo. Nat Immunol. 2009;10:314-24.

35. Gaffen SL, Jain R, Garg AV, Cua DJ. The IL-23-IL-17 immune axis: from mechanisms to therapeutic testing. Nat Rev Immunol. 2014; 14:585-600.

36. Chen Y, Langrish CL, McKenzie B, et al. Anti IL-23 therapy inhibits multiple inflammatory pathways and ameliorates autoimmune encephalomyelitis. J Clin Investig. 2006;116:1317-26.

37. Chackerian AA, Chen SJ, Brodie SJ, et al. Neutralization or absence of interleukin 23 pathway does not compromise immunity to mycobacterial infection. Infect Immun. 2006;74:6092-9.

38. Lieberman LA, Cardillo F, Owyang AM, et al. IL 23 provides a limited meccanism of resistance to acute toxoplasmosis in the absence of IL-12. J Immunol. 2004;173:1887-93.

39. Roncarolo MG, Gregori S, Battaglia M, Bacchetta R, Fleischhauer $\mathrm{K}$, Levings MK. Interleukin-10-secreting type 1 regulatory $\mathrm{T}$ cells in rodents and humans. Immunol Rev. 2006;212:28-50.

40. Xu J, Yang Y, Qiu G, et al. c-Maf regulates IL-10 expression during Th17 polarization. J Immunol. 2009;182(10):6226-36.

41. Leipe J, Grunke M, Dechant C, et al. Role of Th17 cells in human autoimmune arthritis. Arthr Rheumatol. 2010;62(10):2876-85.

42. Chen W, Jin W, Hardegen N, et al. Conversion of peripheral CD4+ CD25- naive $\mathrm{T}$ cells to CD4+CD25+ regulatory $\mathrm{T}$ cells by TGF-beta induction of transcription factor Foxp3. J Exp Med. 2003;198(12):1875-86.

43. Bas M, Adams V, Suvorava T, Niehues T, Hoffmann TK, Kojda G. Nonallergic angioedema: role of bradykinin. Allergy. 2007;10:842-56.

44. Pan ZK, Zuraw BL, Lung CC, Prossnitz ER, Browning DD, Ye RD. Bradykinin stimulates NF-kappaB activation and interleukin 1-beta gene expression in cultured human fibroblasts. J Clin Investig. 1996;98:2042-9.

45. Brovkovych V, Zhang Y, Brovkovych S, Minshall RD, Skidgel RA. A novel pathway for receptor-mediated post-translational activation of inducible nitric oxide synthase. J Cell Mol Med. 2011;15:258-69.

46. Hofman ZL, Relan A, Hack CE. C-rective protein levels in hereditary angioedema. Clin Exp Immunol. 2014;177:280-6.

47. Uzawa A, Mori M, Taniguchi J, Kuwabara S. Modulation of kallikrein/kinin system by the angiotensin-converting enzyme inhibitor alleviates experimental autoimmune encephalomyelitis. Clin Exp Immunol. 2014;178:245-52. 